\title{
CLASSIFICATION OF LEFT INVARIANT HERMITIAN STRUCTURES ON 4-DIMENSIONAL NON-COMPACT RANK ONE SYMMETRIC SPACES
}

\author{
SRDJAN VUKMIROVIĆ, MARIJANA BABIĆ, AND ANDRIJANA DEKIĆ
}

\begin{abstract}
The only 4-dimensional non-compact rank one symmetric spaces are $\mathbb{C} H^{2}$ and $\mathbb{R} H^{4}$. By the classical results of Heintze, one can model these spaces by real solvable Lie groups with left invariant metrics. In this paper we classify all possible left invariant Hermitian structures on these Lie groups, i.e., left invariant Riemannian metrics and the corresponding Hermitian complex structures. We show that each metric from the classification on $\mathbb{C} H^{2}$ admits at least four Hermitian complex structures. One class of metrics on $\mathbb{C} H^{2}$ and all the metrics on $\mathbb{R} H^{4}$ admit 2-spheres of Hermitian complex structures. The standard metric of $\mathbb{C} H^{2}$ is the only Einstein metric from the classification, and also the only metric that admits Kähler structure, while on $\mathbb{R} H^{4}$ all the metrics are Einstein. Finally, we examine the geometry of these Lie groups: curvature properties, self-duality, and holonomy.
\end{abstract}

\section{INTRODUCTION}

Thanks to Heintze [9], it is known that all non-compact rank one symmetric spaces are exactly the hyperbolic spaces

$$
\begin{aligned}
& \mathbb{R} H^{n}=S O_{0}(1, n) / S O(n), \\
& \mathbb{C} H^{n}=S U(1, n) / S(U(1) \times U(n)), \\
& \mathbb{H} H^{n}=S p(1, n) / S p(1) \times S p(n),
\end{aligned}
$$

and Cayley's octonionic plane $\mathbb{O} H^{2}=F_{4(-20)} / \operatorname{Spin}(9)$.

In dimension four this comes down to spaces $\mathbb{C} H^{2}$ and $\mathbb{R} H^{4}$, since the quaternionic hyperbolic line $\mathbb{H} H^{1}$ coincides with $\mathbb{R} H^{4}$. This follows from the isomorphism of Lie groups $S p(1,1) \cong S O(1,4) / \pm$, giving

$$
\mathbb{H} H^{1}=S p(1,1) / S p(1) \times S p(1)=S O(1,4) / S O(4)=\mathbb{R} H^{4} .
$$

From Heintze's papers [9, 10, we also know that a connected homogeneous manifold of non-positive curvature can be represented as a connected solvable Lie

2010 Mathematics Subject Classification. Primary 53B35; Secondary 22E60, 53C20.

Key words and phrases. Non-compact rank one symmetric spaces; Complex hyperbolic plane; Left invariant metrics; Hermitian complex structures.

This research is partially supported within the project 174012 through the Ministry of Education, Science and Technological Development of the Republic of Serbia. 
group with a left invariant metrid 1$] \quad \mathbb{R} H^{4}$ and $\mathbb{C} H^{2}$ are both symmetric spaces of negative sectional curvature, therefore they are solvmanifolds, i.e., they can be represented as connected solvable Lie groups with left invariant metrics. They are exactly the noncompact part in the Iwasawa decomposition of the corresponding isometry groups. We denote these solvable Lie groups by $\mathcal{R H}^{4}$ and $\mathcal{C H}^{2}$, and their Lie algebras by $r h_{4}$ and $c h_{2}{ }^{2}$

In the sequel we classify the Hermitian structures on these groups: all possible non-isometric left invariant Riemannian metrics and Hermitian complex structures in regard to those metrics.

Left invariant Riemannian metrics. We give the classification of non-isometric left invariant Riemannian metrics on the Lie group $\mathcal{C H}^{2}$ in Theorem 2.1. The classification of non-isometric left invariant Riemannian metrics on the Lie group $\mathcal{R H}^{4}$ is presented in Theorem 3.1. These results are partially known (see [6, Table 2, $A_{4,9}^{1}$ for $c h_{2}, A_{4,5}^{1,1}$ for $\left.\left.r h_{4}\right]\right)$.

In dimension three, Milnor [14 gave a complete solution to the more general problem: he used the cross product to classify all left invariant Riemannian metrics on 3-dimensional Lie groups. For the classification of metrics in higher dimensions we use different methods based on Alekseevsky's result that for completely solvable Lie groups the isometry classes of left invariant metrics are the orbits of the automorphism group acting on the space of left invariant metrics (see [1, 2] and Lemmas 1.1, 1.2.

From Jensen's classification of homogeneous Einstein spaces of dimension four [11, we know that $\mathbb{C} H^{2}$ and $\mathbb{R} H^{4}$ are the only non-compact, non-flat, indecomposable 4-dimensional Riemannian homogenous spaces that admit a left invariant Einstein metric. They are also found in [12] within a classification of 4-dimensional Einstein Lie groups. These results are in line with Alekseevsky's conjecture that every non-compact Riemannian Einstein homogenous space is a solvmanifold. A left invariant Riemannian Einstein metric on a given Lie group, if it exists, is unique up to a scaling (see [13] for recent results on Einstein solvmanifolds). Therefore, the Einstein metrics obtained on $\mathcal{R H}^{4}$ and $\mathcal{C H}^{2}$ in [11, 12] are the usual metrics of hyperbolic spaces $\mathbb{R} H^{4}$ and $\mathbb{C} H^{2}$ viewed as symmetric spaces.

Hermitian complex structures. The main result of this paper is a classification of left invariant Hermitian complex structures on $\mathcal{C H}^{2}$ with respect to all non-isometric left invariant Riemannian metrics (Theorem 2.2). Note that for each left invariant metric there exists a 2-dimensional sphere of Hermitian almost complex structures. However, the integrability condition imposes further restrictions, sometimes so strong that there are even-dimensional Lie groups that do not admit any integrable complex structures at all (see [8]). In our case, finding the integrable

\footnotetext{
${ }^{1}$ Note that a simply connected, homogeneous manifold can be isometric to more than one Lie group equipped with a left invariant metric. In other words, non-isomorphic Lie groups might admit left invariant metrics which make them isometric as Riemannian manifolds. For a way to test whether two given solvmanifolds are isometric see [7].

${ }^{2}$ The Lie algebra $c h_{2}$ is denoted by $\mathfrak{d}_{4, \frac{1}{2}}$ in [3] [4, $A_{4,9}^{1}$ in [6], $H_{3}$ in [16], and $\mathbb{R} A \rtimes n$ in [17].
} 
Hermitian structures on $\mathcal{C H}^{2}$ amounts to intersecting the sphere of Hermitian almost complex structures with some non-trivial surfaces of second order coming from the Nijenhuis integrability condition. On $\mathcal{R H}^{4}$ the integrability condition is automatically satisfied, so each metric allows a sphere of Hermitian complex structures (Theorem 3.2).

The classification of left invariant Hermitian complex structures on $\mathcal{C H}^{2}$ is related to many known results of complex geometry of Lie algebras. For example, $c h_{2}$ appears in Barberis's classification of invariant hypercomplex structures on 4-dimensional Lie groups [4. These structures happen to be Hermitian, but not Kählerian (the first case from Table 1). In fact, it was shown in [5] that $c_{2}$ is one of three non-abelian 4-dimensional Lie algebras admitting both the hypercomplex and the para-hypercomplex structure. Snow [18] investigated 4-dimensional solvable simply-connected real Lie groups with commutator subalgebra of dimension less than three. He classified left invariant complex structures with respect to certain subalgebras of the complexification of real Lie algebras. Ovando [16] extended this result to 4-dimensional Lie algebras with 3-dimensional commutator algebra. The $c h_{2}$ case (denoted by $H_{3}$ in [16]) is related to our result in the special case of the left invariant metric associated with an orthonormal base. Specially, Ovando [17] considered spaces $\mathbb{C} H^{2}$ and $\mathbb{R} H^{4}$ modelized as Lie groups and classified all left invariant complex structures with respect to standard metrics (see Remarks 2.2 3.1. In the $r h_{4}$ case she found a sphere of Hermitian complex structures which is consistent with our result.

Outline. Section 1 explains the automorphism group action on the space of left invariant metrics. Basic notation and formulas are introduced here.

In Section 2 we classify Hermitian structures on $\mathcal{C H}^{2}$. The group of automorphisms of the Lie algebra $c h_{2}$ is given by Lemma 2.1. Theorem 2.1 classifies all nonisometric positive definite inner products on $c h_{2}$. They are denoted by $S(p, x, \beta)$, where $p, \beta>0, x \geq 0$ are real parameters, and $p-x^{2}>0$. The corresponding left invariant Riemannian metrics are denoted by $g(p, x, \beta)$. Lemma 2.2 describes the isometric automorphisms of these metrics. In Theorem 2.2 we classify Hermitian complex structures on $\mathcal{C H}^{2}$ with respect to $g(p, x, \beta)$. Their matrix representations are listed in Table 1.

In Section 3 we classify Hermitian structures on $\mathcal{R H}^{4}$. Lemma 3.1 describes the automorphisms of the Lie algebra $r h_{4}$, and Theorem 3.1 classifies non-isometric positive definite inner products $S(\lambda)$. We prove that each of the metrics $g(\lambda)$, corresponding to $S(\lambda)$, has a 2-dimensional sphere of Hermitian complex structures (Theorem 3.2).

Section 4 considers geometry of the metric Lie groups $\mathcal{C H}^{2}$ and $\mathcal{R} \mathcal{H}^{4}$. Curvature properties of $\mathcal{C H}^{2}$ are given in Theorem 4.1. In Theorem 4.2 we show that all metrics $g(p, x, \beta)$ have the full holonomy, except the Kähler metric $g\left(\frac{1}{\beta}, 0, \beta\right)$ whose holonomy group is $U(2)$. Theorem 4.3 shows that all metrics $g(\lambda)$ on $\mathcal{R H}^{4}$ have constant negative sectional curvature and full holonomy group.

We are very grateful to our colleagues Neda Bokan and Tijana Šukilović for many valuable comments and suggestions. 


\section{Preliminaries}

A Lie algebra $\mathfrak{g}$ together with an inner product is called a metric Lie algebra. Every metric Lie algebra defines, by left translations, the unique left invariant metric on the corresponding Lie group $G$, called a metric Lie group. Two metric Lie algebras are isometric if they are isomorphic as vector spaces, and the isomorphism between them preserves curvature tensor and its covariant derivatives. In other words:

Lemma 1.1 (Alekseevsky [2]). Two metric Lie algebras are isometric if and only if the corresponding Riemannian spaces are isometric.

In general, two isometric metric Lie algebras do not have to be isomorphic. However, this does hold for completely solvable Lie algebras.

Lemma 1.2 (Alekseevsky [1, 2]). Isometric completely solvable metric Lie algebras are isomorphic.

Therefore, the isometry classes of different left invariant metrics are precisely the orbits of the automorphism group $\operatorname{Aut}(\mathfrak{g})$ acting on the space of left invariant metrics. Since $\mathcal{C H}^{2}$ and $\mathcal{R} \mathcal{H}^{4}$ are completely solvable groups, this allows us to classify non-isometric metrics using algebraic methods in Theorems 2.1 and 3.1 .

Recall that an almost complex structure on a manifold $M$ is an automorphism $J: T M \rightarrow T M$ satisfying

$$
J^{2}=-I d
$$

If $g$ is a Riemannian metric on $M$, then $J$ is Hermitian if

$$
g(X, Y)=g(J X, J Y), \quad X, Y \in T M .
$$

The space of Hermitian orientation preserving almost complex structures on $2 n$-dimensional vector space with a given positive definite inner product is the symmetric space $S O(2 n) / U(n)$. For $2 n=4$ we have $S O(4) / U(2)=S^{2}$, the two dimensional sphere of the almost complex Hermitian structures.

The Nijenhuis tensor $N$ of an almost complex structure $J$ is a tensor field of rank $(1,2)$ given by

$$
N(X, Y)=[J X, J Y]-[X, Y]-J[J X, Y]-J[X, J Y]
$$

for any $X, Y \in T M$.

If an almost complex structure $J$ is integrable, i.e., its Nijenhuis tensor vanishes identically, then $J$ is a complex structure and $M$ is a complex manifold. In Theorems 2.2 and 3.2 we essentially look for those almost complex Hermitian structures that annihilate the Nijenhuis tensor.

Note that a Lie group admitting a left invariant complex structure may not be a complex Lie group. In order to have the structure of a complex Lie group, the group multiplication has to be holomorphic, or equivalently, the complex structure $J$ has to be ad-invariant.

Let $(M, g)$ be a Riemannian manifold and $\nabla$ its Levi-Civita connection. When the manifold $M$ is a Lie group, Koszul's formula reduces to

$$
2 g\left(\nabla_{X} Y, Z\right)=g([X, Y], Z)-g([Y, Z], X)+g([Z, X], Y)
$$


for any left invariant vector fields $X, Y, Z$.

We define the Riemann curvature operator $R(X, Y): T_{p} M \rightarrow T_{p} M, p \in M$, by

$$
R(X, Y)=\nabla_{X} \nabla_{Y}-\nabla_{Y} \nabla_{X}-\nabla_{[X, Y]} X
$$

for any vector fields $X, Y$ on $T_{p} M$. The Ricci curvature tensor is defined by

$$
\rho(X, Y)=\operatorname{Tr}(Z \mapsto R(Z, X) Y) .
$$

A manifold $(M, g)$ is Einstein if $\rho$ is proportional to the metric $g$. The scalar curvature $\tau$ is the trace of the Ricci tensor with respect to the metric $g$. If $(X, Y)$ is a basis of a 2-dimensional plane $\pi \subset T_{p} M$ then

$$
K(\pi):=\frac{g(R(X, Y) Y, X)}{g(X, X) g(Y, Y)-g(X, Y)^{2}}
$$

is a sectional curvature in the plane $\pi$. If $M$ is endowed with a Hermitian complex structure $J$, then for a unit vector $X \in T_{p} M$, vectors $X$ and $J X$ form an orthonormal basis of the $J$-invariant plane $\pi$ and $K(\pi)$ is called a holomorphical sectional curvature.

The curvature operators $R(X, Y)$ satisfy $g(R(X, Y) Z, W)=-g(R(X, Y) W, Z)$ and therefore belong to the algebra of skew-symmetric endomorphisms $s o(g)$. We identify the skew-symmetric endomorphisms $s o(g)$ with 2 -vectors $\Lambda^{2} T_{p} M$ by the formula

$$
(X \wedge Y)(Z):=g(Z, Y) X-g(Z, X) Y,
$$

for any $Z \in T_{p} M$. Since $R(X, Y)=-R(Y, X)$, the curvature tensor can be regarded as an operator on the space of 2 -vectors

$$
R: \Lambda^{2} T_{p} M \rightarrow \Lambda^{2} T_{p} M \cong s o(g), \quad R(X \wedge Y):=R(X, Y) .
$$

A Riemannian metric on $T_{p} M$ naturally induces a positive definite inner product $\langle\cdot, \cdot\rangle$ on the space of $k$-vectors $\Lambda^{k} T_{p} M$ by

$$
\left\langle X_{1} \wedge \cdots \wedge X_{k}, Y_{1} \wedge \cdots \wedge Y_{k}\right\rangle:=\operatorname{det}\left(g\left(X_{i}, Y_{j}\right)\right) .
$$

The operator $R: \Lambda^{2} T_{p} M \rightarrow \Lambda^{2} T_{p} M$ is symmetric with respect to the inner product $\langle\cdot, \cdot\rangle$ on $\Lambda^{2} T_{p} M$.

If $M$ is 4-dimensional and $\omega \in \Lambda^{4} T_{p} M$ is the unit vector, a Hodge star operator * : $\Lambda^{2} T_{p} M \rightarrow \Lambda^{2} T_{p} M$ is defined by the relation

$$
\alpha \wedge * \beta=\langle\alpha, \beta\rangle \omega
$$

It is symmetric with respect to the inner product $\langle\cdot, \cdot\rangle$ and satisfies $*^{2}=I d$. Therefore, it induces an orthogonal splitting

$$
\Lambda^{2}=\Lambda_{+}^{2} \oplus \Lambda_{-}^{2}
$$

of the 6 -dimensional space $\Lambda^{2}$ into the 3 -dimensional \pm 1 eigenspaces of $*$.

With respect to that splitting, the curvature tensor has the well known decomposition

$$
R=\frac{\tau}{12} I_{6}+\left(\begin{array}{cc}
W_{+} & B \\
B^{*} & W_{-}
\end{array}\right)
$$


where $\tau$ denotes the scalar curvature, $W_{ \pm}$is the Weyl conformal tensor, and $B$ is the traceless Ricci tensor. We say that $(M, g)$ is self-dual (resp. anti-self-dual) if $W_{+}=0$ (resp. $W_{-}=0$ ) and conformally flat if $W_{+}=0=W_{-}$. If either of $W_{ \pm}$ vanishes we say that $M$ is half-conformally flat. The manifold is Einstein if and only if $B=0$.

\section{Classification of left invariant Hermitian structures on $\mathcal{C H}^{2}$}

Consider the Iwasawa decomposition, $S U(1,2)=K A N$, of the semisimple group of isometries of $\mathbb{C} H^{2}$. The compact part is isomorphic to $U(2)$, the nilpotent part $N$ is the Heisenberg group $H_{3}$, and the abelian part is 1-dimensional. The completely solvable group $\mathcal{C H}^{2}=A N$ acts simply transitively on the complex hyperbolic plane $\mathbb{C} H^{2}$ and gives it a structure of a Lie group in a way that its Kähler metric is left invariant. Its Lie algebra $c h_{2}$ is spanned by vectors $X, Y, Z, W$ with nonzero commutators:

$$
c h_{2}:[X, Y]=\frac{1}{2} Y, \quad[X, Z]=\frac{1}{2} Z, \quad[X, W]=W, \quad[Z, Y]=W .
$$

$c h_{2}$ is a semidirect product of the nilpotent Heisenberg ideal spanned by $Y, Z, W$ and the 1-dimensional center spanned by $X$.

$\mathcal{C H}^{2}$ is a solvmanifold in a trivial way, i.e., it acts on itself isometrically by left translations. Equipped with the Kähler left invariant metric, $\mathcal{C H}^{2}$ is the complex hyperbolic plane $\mathbb{C} H^{2}$ in the usual sense.

Lemma 2.1. The automorphism group of the Lie algebra $\mathrm{ch}_{2}$ is

$$
\operatorname{Aut}\left(c h_{2}\right)=\left\{\left(\begin{array}{cccc}
1 & 0 & 0 & 0 \\
u_{1} & a & b & 0 \\
u_{2} & c & d & 0 \\
e & v_{1} & v_{2} & \lambda
\end{array}\right) \mid\left(\begin{array}{l}
u_{1} \\
u_{2}
\end{array}\right)=\frac{1}{2 \lambda}\left(\begin{array}{cc}
-b & a \\
-d & c
\end{array}\right)\left(\begin{array}{l}
v_{1} \\
v_{2}
\end{array}\right), \lambda=a d-b c\right\} \text {. }
$$

Theorem 2.1. All non-isometric positive definite inner products on $\mathrm{ch}_{2}$, in some basis with Lie algebra commutators (10), are represented by the matrices

$$
S(p, x, \beta)=\left(\begin{array}{cccc}
p & x & 0 & 0 \\
x & 1 & 0 & 0 \\
0 & 0 & 1 & 0 \\
0 & 0 & 0 & \beta
\end{array}\right)
$$

where $x \geq 0, \beta>0, p-x^{2}>0$.

Proof. Two different inner products on $c_{2}$ define two metric Lie algebras on the same space. If the inner products are represented by symmetric positive definite matrices $S$ and $F^{T} S F, F \in \operatorname{Aut}\left(c h_{2}\right)$, those metric Lie algebras are isomorphic, and therefore they are isometric. The converse statement follows from Lemma 1.2. Thus, non-isometric left invariant inner products correspond to the orbits of the group $\operatorname{Aut}\left(c h_{2}\right)$ acting on the space of positive definite symmetric matrices. Lengthy calculations similar to the proof of Theorem $3.1\left(r h_{4}\right.$ case $)$ show that these orbits are represented by the matrices of the form $S(p, x, \beta)$. 
A left invariant metric on a Lie group is uniquely determined by an inner product on its Lie algebra. We denote by $g(p, x, \beta)$ the left invariant Riemannian metric on $\mathcal{C H}^{2}$ corresponding to the inner product $S(p, x, \beta)$. From Lemma 1.1 it follows that the isometry classes of the metrics on $\mathcal{C H}^{2}$ are the orbits of the automorphism group Aut $\left(\mathcal{C H}^{2}\right)$ acting on the space of left invariant metrics. Therefore, Theorem 2.1 gives us the classification of all non-isometric left invariant metrics on $\mathcal{C H}^{2}$.

Lemma 2.2. The group of isometric automorphisms of ch $\mathrm{c}_{2}$ with the inner product $S(p, x, \beta)$ is isomorphic to the discrete group $\mathbb{Z}_{2}$, if $x>0$, and to the orthogonal group $O(2)$, if $x=0$.

Proof. The matrix of an automorphism $F$ is of the form (11). It is an isometry if $F^{T} S F=S$ is satisfied.

If $x>0$, then $F$ is either the identity matrix or $F=\operatorname{diag}(1,1,-1,-1)$.

$$
\text { If } x=0 \text {, then } F=\left(\begin{array}{cccc}
1 & 0 & 0 & 0 \\
0 & \cos \phi & -\lambda \sin \phi & 0 \\
0 & \sin \phi & \lambda \cos \phi & 0 \\
0 & 0 & 0 & \lambda
\end{array}\right), \lambda= \pm 1 \text {. }
$$

Now we classify the left invariant Hermitian complex structures on $\mathcal{C H}^{2}$ with respect to all non-isometric left invariant Riemannian metrics $g(p, x, \beta)$.

Theorem 2.2. All left-invariant Hermitian complex structures on the metric Lie group $\left(\mathcal{C H}^{2}, g\right)$ corresponding to the metric Lie algebra ch $_{2}$ with the inner product $S(p, x, \beta)$ are:

1a) for $x=0,1-4 p \beta=0$, the 2-dimensional sphere of structures $\left\{J_{s, r, t} \mid s^{2}+\right.$ $\left.r^{2}+t^{2}=1\right\}$

1b) for $x=0,1-4 p \beta \neq 0$, \& structures $J_{K \pm}, J_{N K \pm}$;

2a) for $x>0,1-4 p \beta=0$, 4 structures $J_{1 \pm}, J_{2 \pm}$;

2b) for $x>0,1-4 p \beta \neq 0$, $\operatorname{det} S \neq 4 p^{2} \beta^{2}$, 4 structures $J_{3 \pm}, J_{4 \pm}$;

2c) for $x>0,1-4 p \beta \neq 0$, $\operatorname{det} S=4 p^{2} \beta^{2}$, 4 structures $J_{5 \pm}, J_{6 \pm}$.

Matrices of these structures and their isometric isomorphism classes are given in Table 1 .

Proof. Let $J=\left(a_{i j}\right)$ denote the matrix representing the complex structure $J$ in a basis with commutators 10 . Since $J$ is a Hermitian complex structure, it imposes certain constraints on the entries of the matrix $J=\left(a_{i j}\right)$.

The condition (2) that the structure $J$ is Hermitian, is equivalent to

$$
J^{T} S+S J=0
$$




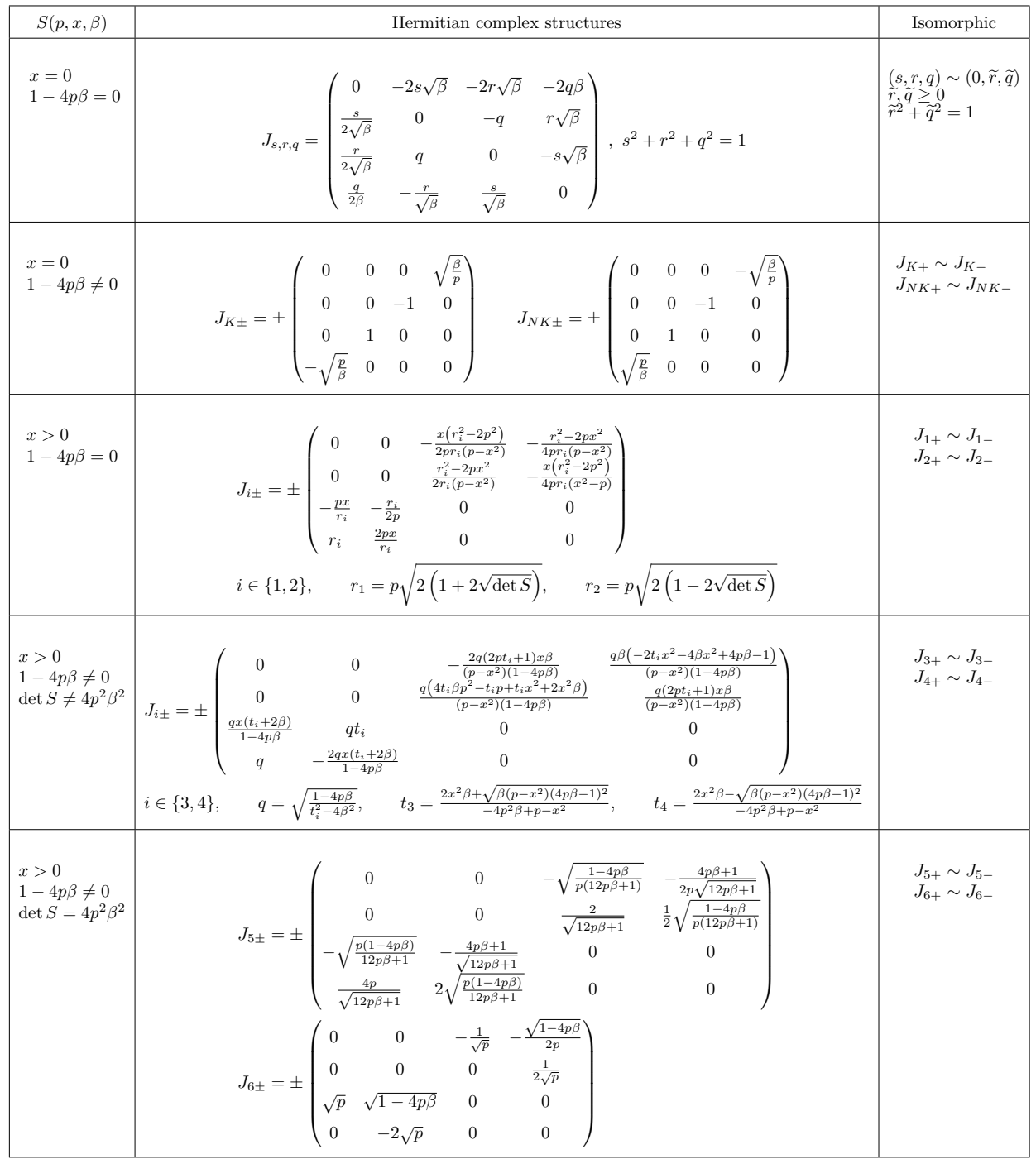

TABLE 1. Hermitian complex structures on $c h_{2}$ with inner product $S(p, x, \beta)$.

which implies the following linear relations:

$$
\begin{aligned}
& a_{33}=0, \quad a_{44}=0, \quad a_{22}=-a_{12} x, \\
& a_{34}=-a_{43} \beta, \quad a_{11}=a_{12} x, \quad a_{21}=-a_{12} p, \\
& a_{13}=\frac{a_{31}-a_{32} x}{x^{2}-p}, \quad a_{23}=\frac{-a_{32} p+a_{31} x}{p-x^{2}}, \\
& a_{14}=\frac{-a_{41} \beta+a_{42} x \beta}{p-x^{2}}, \quad a_{24}=\frac{-a_{42} p \beta+a_{41} x \beta}{p-x^{2}} .
\end{aligned}
$$


The condition that $J$ is a complex structure is equivalent to the system of quadratic equations

$$
N(X, Y)=0, \quad J^{2}=-I d,
$$

where the Nijenhuis tensor $N$ is given by $(3)$. These quadratic constraints lead us to discuss the following cases:

Case 1a. $x=0,1-4 p \beta=0$.

Conditions (13) are now equivalent to

$$
4 a_{31}^{2} \beta+4 a_{41}^{2} \beta^{2}+a_{43}^{2} \beta=1 .
$$

Denoting

$$
r=2 a_{31} \sqrt{\beta}, \quad s=a_{43} \sqrt{\beta}, \quad t=2 a_{41} \beta
$$

we have the sphere of complex structures $J_{r, s, t}$, with $r^{2}+s^{2}+t^{2}=1$, listed in Table 1

Case 1b. $x=0,1-4 p \beta \neq 0$.

Conditions (13), together with the regularity of $J$, are now equivalent to

$$
a_{43}=0, \quad a_{12}=0, \quad a_{42}=0, \quad a_{31}=0, \quad a_{32}^{2}=1, \quad a_{41}^{2}=\frac{p}{\beta} .
$$

Consequently, in this case we have four complex structures $J_{K \pm}, J_{N K \pm}$, listed in Table 1

Case 2. $x>0$.

From conditions (13) and the regularity of $J$ it follows that

$$
\begin{aligned}
& a_{43}=0, \quad a_{12}=0, \\
& a_{31}=a_{32} x-2 a_{42} p \beta+2 a_{41} x \beta, \\
& a_{32}^{2}+\frac{\left(a_{42}+2 a_{32} x\right)\left(a_{42} p-a_{41} x\right) \beta}{p-x^{2}}-1=0, \\
& a_{32}^{2}+\frac{4\left(a_{42} p-a_{41} x\right)^{2} \beta^{2}}{p-x^{2}}-1=0 .
\end{aligned}
$$

Case 2a. $x>0,1-4 p \beta=0$.

From conditions 15 and 16 it follows that $a_{32}=-\frac{a_{41}}{2 p}$. From the relation (14) we get $a_{31}=-\frac{a_{42}}{2}$. Conditions 13 are now equivalent to

$$
\begin{gathered}
a_{41} a_{42}=2 p x, \\
a_{41}^{2}+a_{42}^{2} p=4 p^{2} .
\end{gathered}
$$

Now we consider the system (17), (18) with respect to coordinates $\left(a_{41}, a_{42}\right)$. Since $p \neq 0, x \neq 0$, the equation (17) is always a hyperbola, and the equation (18) is an ellipse. One can check that these curves always intersect in 4 points given by

$$
a_{41}= \pm \sqrt{2(1 \pm 2 \sqrt{\operatorname{det} S})}, \quad a_{42}=\frac{2 p x}{a_{41}} .
$$

All four possible values of $a_{41}$ are real under the given conditions. Denoting

$$
r_{1}=p \sqrt{2(1+2 \sqrt{\operatorname{det} S})}, \quad r_{2}=p \sqrt{2(1-2 \sqrt{\operatorname{det} S})},
$$


we have matrices of the corresponding complex structures $J_{1 \pm}$ and $J_{2 \pm}$ given in Table 1 .

Case $x>0,1-4 p \beta \neq 0$.

From conditions $(15)$ and $(16)$ and the regularity of the matrix $J$ it follows that

$$
a_{42}=\frac{2\left(a_{32} x+2 a_{41} x \beta\right)}{4 p \beta-1} .
$$

Conditions 13 are now equivalent to

$$
\begin{gathered}
a_{32}^{2}-4 a_{41}^{2} \beta^{2}+4 p \beta-1=0, \\
\left(4 \beta p^{2}-p+x^{2}\right) a_{32}^{2}+4 a_{32} a_{41} \beta x^{2}+\left(\beta-4 \beta^{2} p+4 \beta^{2} x^{2}\right) a_{41}^{2}=0 .
\end{gathered}
$$

The equation 19 with variables $a_{32}$ and $a_{41}$ represents a hyperbola. Notice that the determinant of the quadratic form $(20)$ equals $-(1-4 p \beta)^{2} \operatorname{det} S$. It is strictly negative, so the equation 20 represents two lines intersecting at the origin. Therefore, the solution of the system above is the intersection of a hyperbola and two lines. The coefficient of the term $a_{32}^{2}$ in $(20)$ equals $\frac{1}{\beta}\left(4 p^{2} \beta^{2}-\operatorname{det} S\right)$ which leads us to consider the following cases:

Case 2b. $x>0,1-4 p \beta \neq 0, \quad \operatorname{det} S \neq 4 p^{2} \beta^{2}$.

In this case, $a_{41} \neq 0$. We put $a_{32}=t a_{41}$ and from 20 we get

$$
a_{41}=\sqrt{\frac{1-4 p \beta}{t^{2}-4 \beta^{2}}}, \quad t_{ \pm}=\frac{2 x^{2} \beta \pm \sqrt{\beta\left(p-x^{2}\right)(4 p \beta-1)^{2}}}{-4 p^{2} \beta+p-x^{2}} .
$$

The corresponding complex structures $J_{3 \pm}$ and $J_{4 \pm}$ are given in Table 1 . We use the notation $t_{3}=t_{+}, t_{4}=t_{-}, q=a_{41}$.

Case 2c. $x>0,1-4 p \beta \neq 0, \operatorname{det} S=4 p^{2} \beta^{2}$.

In this case equation 20 becomes

$$
a_{41} \beta(1-4 p \beta)\left(4 a_{32} p+4 a_{41} p \beta+a_{41}\right)=0 .
$$

The only two options, $a_{41} \neq 0$ and $a_{41}=0$, give structures $J_{5 \pm}$ and $J_{6 \pm}$ respectively. This covers all the cases, so all complex structures have been found.

If two structures $J$ and $F^{-1} J F$ are related by an isometric automorphism from Lemma 2.2 they determine the same geometry. We call them isomorphic and they are listed in Table 1

Remark 2.1. The sphere of complex structures $\left\{J_{s, r, t}\right\}$ from Theorem 2.2 was found by Barberis [4] within a classification of hypercomplex structures on 4-dimensional Lie groups. Namely, the structures $I=J_{1,0,0}, J=J_{0,1,0}, K=J_{0,0,1}$ constitute a hypercomplex structure $(I, J, K)$, i.e., they satisfy $I J=K=-J I$. It is interesting that a hypercomplex structure on a 4-dimensional manifold determines a conformally unique Hermitian metric. On the Lie group $\mathcal{C H}^{2}$ that metric is $g\left(\frac{1}{4 \beta}, 0, \beta\right), \beta>0$ from the classification. 
Remark 2.2. Ovando [16, 17] found three classes of left invariant complex structures with respect to certain subalgebras of the complexification of the real Lie algebra $c h_{2}$. Considering an orthonormal basis (thus fixing one left invariant metric) she noted that one of those classes contains the standard Kähler metric on $\mathbb{C} H^{2}$. This case corresponds to $J_{K \pm}$ from our classification in the special case of the left invariant metric $g(1,0,1)$. The second class from [16] corresponds to $J_{N K \pm}$ with the same metrics, and complex structures from the third class are not Hermitian.

\section{Classification of left invariant Hermitian structures on $\mathcal{R H}^{4}$}

If $S O(1,4)=K A N$ is the Iwasawa decomposition of the group of isometries of $\mathbb{R} H^{4}$, then the compact part is $S O(4)$, the abelian part is $\mathbb{R}^{3}$, and the nilpotent part is 1-dimensional. The Lie group $\mathcal{R H}^{4}=A N$, acting simply transitively on the real hyperbolic space $\mathbb{R} H^{4}$, has the Lie algebra $r h_{4}$ spanned by vectors $e_{1}, e_{2}, e_{3}, e_{4}$ with nonzero commutators:

$$
r h_{4}:\left[e_{4}, e_{k}\right]=e_{k}, \quad k=1,2,3 .
$$

It is a two step nilpotent algebra with a 3 -dimensional commutator subalgebra spanned by $e_{1}, e_{2}, e_{3}$.

Lemma 3.1. The automorphism group of $r h_{4}$ is

$$
\operatorname{Aut}\left(r h_{4}\right)=\left\{\left(\begin{array}{ll}
A & b \\
0 & 1
\end{array}\right) \mid A \in G l_{3}(\mathbb{R}), v \in \mathbb{R}^{3}\right\} .
$$

This group is isomorphic to the group $\mathrm{Aff}_{3}(\mathbb{R})$ of affine transformations of $\mathbb{R}^{3}$.

Theorem 3.1. All non-isometric positive definite inner products on $\mathrm{rh}_{4}$, in some basis with Lie algebra commutators (21), are represented by the matrices

$$
S(\lambda)=\left(\begin{array}{cc}
I_{3} & 0 \\
0 & \lambda
\end{array}\right)
$$

where $\lambda>0$.

Proof. Thanks to Alekseevsky's results (Lemmas 1.1 and 1.2, describing nonisometric inner products on $r h_{4}$ is equivalent to finding equivalence classes of positive definite symmetric matrices $S$ under the equivalence relation $S \sim F^{T} S F$, where $F=F(A, b)$ is an automorphism of the form $(22)$ (i.e., finding orbits of the automorphism group). If $S$ is any symmetric positive definite $4 \times 4$ matrix, we can write it in a block matrix form $S=\left(\begin{array}{cc}\bar{S} & v \\ v^{T} & \bar{\lambda}\end{array}\right)$, where $\bar{S}$ is a symmetric positive definite $3 \times 3$ matrix, $v \in \mathbb{R}^{3}, \bar{\lambda} \in \mathbb{R}$. Choosing the automorphism $F_{1}\left(I_{3},-\bar{S}^{-1} v\right), S$ gets simplified to $S_{1}=F_{1}^{T} S F_{1}=\left(\begin{array}{cc}\bar{S} & 0 \\ 0 & \lambda\end{array}\right)$. Now we choose automorphism $F_{2}(A, 0)$, where $A$ is a matrix diagonalizing $\bar{S}$. The resulting matrix $S_{2}=F_{2}^{T} S F_{2}=\left(\begin{array}{cc}I_{3} & 0 \\ 0 & \lambda\end{array}\right)$ is the simplest representative of an orbit of the automorphism group acting on the space of positive definite symmetric matrices. 
Every positive definite inner product $S(\lambda)$ gives rise to a unique left invariant Riemannian metric $g(\lambda)$ by left translations, so the previous theorem describes all non-isometric left invariant Riemannian metrics on $\mathcal{R H}^{4}$.

Theorem 3.2. Every left invariant metric $g(\lambda)$ on $\mathcal{R H}^{4}$, determined by the inner products $S(\lambda), \lambda>0$ on $r_{4}$, admits a 2-dimensional sphere of Hermitian complex structures

$$
\left(\begin{array}{cccc}
0 & -\alpha & -\beta & -\gamma \sqrt{\lambda} \\
\alpha & 0 & -\gamma & \beta \sqrt{\lambda} \\
\beta & \gamma & 0 & -\alpha \sqrt{\lambda} \\
\frac{\gamma}{\sqrt{\lambda}} & -\frac{\beta}{\sqrt{\lambda}} & \frac{\alpha}{\sqrt{\lambda}} & 0
\end{array}\right), \quad \alpha^{2}+\beta^{2}+\gamma^{2}=1 .
$$

Proof. Direct calculation shows that conditions of integrability (Nijenhuis) are a consequence of conditions $J^{2}=-I d$ and $J$ being Hermitian.

Remark 3.1. With respect to the standard metric $g(1)$, Ovando [17] found a 2 -sphere of Hermitian complex structures on $r h_{4}$ which is consistent with the previous result.

\section{Left inVARIANt GeOMETRY of Metric Lie groups $\mathcal{C H}^{2}$ AND $\mathcal{R} \mathcal{H}^{4}$}

Now we are ready to prove the theorems that describe curvature properties of left invariant metrics $g(p, x, \beta)$ on $\mathcal{C H}^{2}$, and of left invariant metrics $g(\lambda)$ on $\mathcal{R H}^{4}$.

\section{Theorem 4.1.}

i) For all left invariant Riemannian metrics $g(p, x, \beta), \mathcal{C H}^{2}$ has a constant negative scalar curvature

$$
\tau=-\frac{11+p \beta}{2\left(p-x^{2}\right)} .
$$

ii) Among $g(p, x, \beta)$, the unique Einstein metric is $g\left(\frac{1}{\beta}, 0, \beta\right), \beta>0$. Moreover, it is the unique metric that admits a Kähler structure ( $J_{K \pm}$ in classification). $\mathcal{C H}^{2}$ with this metric is a solvmanifold with constant holomorphic sectional curvature $-\beta$ and it is isometric to the complex hyperbolic plane $\mathbb{C} H^{2}$.

iii) $g\left(\frac{1}{4 \beta}, 0, \beta\right)$ and $g\left(\frac{1}{\beta}, 0, \beta\right)$ are the only half-conformally flat metrics on $\mathcal{C H}^{2}$.

Proof. Since we work with left invariant basis, we have a natural identification $T_{p} \mathcal{C H}^{2} \cong c h_{2}$ for any $p \in \mathcal{C H}^{2}$. Let $(X, Y, Z, W)$ be a basis of $c h_{2}$ with commutators of the form $(10)$. In that basis, the metric $g(p, x, \beta)$ is represented by the matrix $S=S(p, x, \beta)$ of the form $(12)$.

The Levi-Civita connection can be calculated by Koszul's formula (4). Using the fact that $\nabla$ is torsion-free, $\nabla_{X_{1}} X_{2}-\nabla_{X_{2}} X_{1}=\left[X_{1}, X_{2}\right]$, it follows that all nonzero 
covariant derivatives are determined by

$$
\begin{aligned}
& \nabla_{X} X=\frac{x}{2\left(p-x^{2}\right)}(x X-p Y), \quad \nabla_{X} Y=\frac{x}{2\left(p-x^{2}\right)}(X-x Y), \\
& \nabla_{Y} Y=\frac{1}{2\left(p-x^{2}\right)}(X-x Y), \quad \nabla_{Y} Z=-\frac{1}{2} W, \quad \nabla_{Y} W=\frac{\beta}{2} Z, \\
& \nabla_{Z} Z=\frac{1}{2\left(p-x^{2}\right)}(X-x Y), \quad \nabla_{Z} W=\frac{\beta}{2\left(p-x^{2}\right)}(x X-p Y), \\
& \nabla_{W} W=\frac{\beta}{p-x^{2}}(X-x Y) .
\end{aligned}
$$

Note that the 2-dimensional submanifold whose tangent space is spanned by left invariant vector fields $X$ and $Y$ is totally geodesic. Using formula (5) we obtain the curvature operator $R: \Lambda^{2} \operatorname{ch}_{2} \rightarrow \Lambda^{2} \operatorname{ch}_{2}$ :

$$
\begin{aligned}
R(X \wedge Y) & =-\frac{1}{4\left(p-x^{2}\right)}\left(X \wedge Y+\left(p-x^{2}\right) Z \wedge W\right), \\
R(X \wedge Z) & =-\frac{1}{4\left(p-x^{2}\right)}(X \wedge Y+2 x X \wedge W-p Y \wedge W), \\
R(X \wedge W) & =-\frac{1}{4\left(p-x^{2}\right)}(3 x \beta X \wedge Z+4 X \wedge W+2 \beta Y \wedge Z-2 x Y \wedge W), \\
R(Y \wedge Z) & =\frac{1}{4\left(p-x^{2}\right)}(3 x \beta X \wedge Z+2 X \wedge W-(1+3 p \beta) Y \wedge Z-3 x Y \wedge W), \\
R(Y \wedge W) & =\frac{1}{4\left(p-x^{2}\right)}(\beta X \wedge Z-x \beta X \wedge W-2 x \beta Y \wedge Z+(p \beta-2) Y \wedge W), \\
R(Z \wedge W) & =-\frac{1}{4\left(p-x^{2}\right)}(\beta X \wedge Y-(p \beta-2) Z \wedge W) .
\end{aligned}
$$

Using formula (6) we obtain the Ricci tensor matrix in the basis $(X, Y, Z, W)$ :

$$
\rho=\frac{1}{p-x^{2}}\left(\begin{array}{cccc}
\frac{x^{2}-3 p}{2} & -x & 0 & 0 \\
-x & -\frac{p \beta-x^{2} \beta+2}{2} & 0 & 0 \\
0 & 0 & -\frac{p \beta+2}{2} & \frac{-5 x \beta}{4} \\
0 & 0 & \frac{-5 x \beta}{4} & \frac{\beta(p \beta-4)}{2}
\end{array}\right) .
$$

The scalar curvature is then

$$
\tau=-\frac{11+p \beta}{2\left(p-x^{2}\right)} .
$$

Since $p, \beta>0$ and $p-x^{2}>0$, the scalar curvature is negative. This proves part i) of the theorem.

The condition $g(p, x, \beta)$ is Einstein, i.e., $\rho=\lambda g$ for some $\lambda \in \mathbb{R}$, is according to (12) and 25 equivalent to

$$
x=0, \quad p \beta=1, \quad \text { and } \quad \lambda=-\frac{3}{2 \beta} .
$$


Therefore, the only Einstein left invariant metric on $\mathcal{C H}^{2}$ is $g\left(\frac{1}{\beta}, 0, \beta\right)$. For the complex structure $J_{K \pm}$ from Table 1 direct calculation shows $\nabla_{V} J_{K \pm}=0$ for any $V \in c h_{2}$. Thus, $\left(\mathcal{C H}^{2}, g\left(\frac{1}{\beta}, 0, \beta\right), J_{K \pm}\right)$ is a Kähler manifold with constant holomorphic sectional curvature $-\beta$, so it is isometric to the complex hyperbolic plane $\mathbb{C} H^{2}$. Note that $g\left(\frac{1}{\beta}, 0, \beta\right)$ with complex structure $J_{N K \pm}$ is not Kählerian, but also has a constant holomorphic sectional curvature $-\beta$.

We check case-by-case structures from Table 1 to prove that no metric other than $g\left(\frac{1}{\beta}, 0, \beta\right)$ admits a Kählerian structure. This proves part ii).

To prove iii) we introduce an orthonormal basis of $c h_{2}$ with respect to the inner product $S(p, x, \beta)$

$$
e_{1}=\frac{1}{\sqrt{p-x^{2}}} X-\frac{x}{\sqrt{p-x^{2}}} Y, \quad e_{2}=Y, \quad e_{3}=Z, \quad e_{4}=\frac{1}{\sqrt{\beta}} .
$$

The basis $e_{i} \wedge e_{j}, i<j$, is an orthonormal basis of $\Lambda^{2} c h_{2}$, and $\omega=e_{1} \wedge e_{2} \wedge e_{3} \wedge e_{4}$ is a unit vector that spans $\Lambda^{4} c h_{2}$. Further lengthy calculations show that

$$
\Lambda_{ \pm}^{2}=\mathcal{L}\left(\frac{1}{\sqrt{2}}\left(e_{1} \wedge e_{2} \pm e_{3} \wedge e_{4}\right), \frac{1}{\sqrt{2}}\left(e_{1} \wedge e_{3} \mp e_{2} \wedge e_{4}\right), \frac{1}{\sqrt{2}}\left(e 1 \wedge e 4 \pm e_{2} \wedge e_{3}\right)\right)
$$

are orthonormal bases of \pm 1 eigenspaces $\Lambda_{ \pm}^{2}$ of the Hodge operator $*$ defined by (8). One can use $R: \Lambda^{2} \rightarrow \Lambda^{2}$ given by (24) with respect to the basis (26) to obtain the decomposition (9). If $x \neq 0$ then $W_{ \pm} \neq 0$, so a metric cannot be half-conformally flat. If $x=0$ we obtain

$$
W_{ \pm}=\frac{1 \pm 3 \sqrt{p \beta}+2 p \beta}{12 p} \operatorname{diag}(1,1,-2) .
$$

We see that $W_{-}$vanishes when $p \beta=1$ or $4 p \beta=1$, so iii) is proved.

Remark 4.1. Milnor showed that the scalar curvature of a non-flat left invariant Riemannian metric on a solvable Lie group is strictly negative ([14, Theorem 3.1]). None of $g(p, x, \beta)$ on $\mathcal{C H}^{2}$ is flat and this is consistent with our result.

Theorem 4.2. All left invariant metrics $g(p, x, \beta)$ on $\mathcal{C H}^{2}$ have the full holonomy group, except the Kähler metric $g\left(\frac{1}{\beta}, 0, \beta\right)$, whose holonomy group is isomorphic to $U(2)$.

Proof. The infinitesimal holonomy algebra is spanned by curvature operators $R(\cdot, \cdot)$ and their covariant derivatives $\nabla^{k} R$ of all degrees $k \in \mathbb{N}$. The corresponding uniquely determined connected subgroup of $G L(4, \mathbb{R})$ is called the infinitesimal holonomy group. Since a Lie group is an analytic manifold, its restricted, local and infinitesimal holonomy groups coincide (see [15]). Moreover, $\mathcal{C H}^{2}$ is simply connected, therefore, the holonomy group is the same as the local holonomy group.

In the $p \beta=1, x=0$ case the metric is symmetric. Hence, $\nabla R=0$ and the holonomy algebra is $u(2)$ spanned only by curvature operators. In all other cases, direct but lengthy calculations show that the holonomy algebra is isomorphic to so(4) and is spanned by curvature operators and their first covariant derivatives. 
We may now conclude that in the $p \beta=1, x=0$ case the holonomy group is $U(2)$. In all other cases, the metric $g$ has the full holonomy group.

Corollary 4.1. All left invariant metrics $g(p, x, \beta)$ on $\mathcal{C H}^{2}$ are indecomposable.

Now we consider the geometry of $\mathcal{R H}^{4}$.

Theorem 4.3. All left invariant Riemannian metrics $g(\lambda)$ on $\mathcal{R H}^{4}$ are Einstein. They have constant negative sectional curvature $-\frac{1}{\lambda}$, constant scalar curvature $-\frac{12}{\lambda}$, and the holonomy group is full.

Proof. From Koszul's formula for left invariant vector fields (4), it follows that all nonzero covariant derivatives are determined by

$$
\nabla_{e_{1}} e_{4}=-e_{1}, \quad \nabla_{e_{2}} e_{4}=-e_{2}, \quad \nabla_{e_{3}} e_{4}=-e_{3}, \quad \nabla_{e_{1}} e_{1}=\nabla_{e_{2}} e_{2}=\nabla_{e_{3}} e_{3}=\frac{e_{4}}{\lambda}
$$

Further calculation brings us a nice expression for the curvature operator $R: \Lambda^{2} r h_{4} \rightarrow \Lambda^{2} r h_{4}$ given on the basis of the space of 2-vectors $\Lambda^{2} r h_{4}$ :

$$
R\left(e_{i} \wedge e_{j}\right)=-\frac{1}{\lambda} e_{i} \wedge e_{j}, \quad 1 \leq i<j \leq 4 .
$$

From definition (7) and the previous formula, it follows that the sectional curvature is

$$
K(\pi)=-\frac{1}{\lambda},
$$

for any 2-dimensional plane $\pi \subset T_{p} \mathcal{R H}^{4}$. Using (6) we find the matrix of the Ricci tensor: $\rho=\operatorname{diag}\left(-\frac{3}{\lambda},-\frac{3}{\lambda},-\frac{3}{\lambda},-3\right)$. Since $\rho$ is proportional to the inner product matrix (23), the corresponding left invariant metric $g(\lambda)$ is Einstein, and the scalar curvature is $\tau=-\frac{12}{\lambda}$. The holonomy algebra is 6 -dimensional, hence $g(\lambda)$ has the full holonomy group.

\section{REFERENCES}

[1] D. V. Alekseevsky, The conjugacy of polar decompositions of Lie groups, Mat. Sbornik (N.S.) 84 (126) (1971), 14-26. MR 0277662

[2] D. V. Alekseevsky, Homogeneous Riemannian spaces of negative curvature, Mat. Sbornik (N.S.) 96 (138) (1975), 93-117, 168. MR 0362145

[3] A. Andrada, M. L. Barberis, I. G. Dotti, G. P. Ovando, Product structures on four dimensional solvable Lie algebras, Homology Homotopy Appl. 7 (2005), no. 1, 9-37. MR 2138348.

[4] M. L. Barberis, Hypercomplex structures on four-dimentional Lie groups, Proc. Amer. Math. Soc. 125 (1997), no. 4, 1043-1054. MR 1353375

[5] N. Blažić, S. Vukmirović, Para-hypercomplex structures on a four-dimensional Lie group, Contemporary geometry and related topics, 41-56, World Sci. Publ., River Edge, NJ, 2004. MR 2070865

[6] T. Christodoulakis, G.O. Papadopoulos, A. Dimakis, Automorphisms of real fourdimensional Lie algebras and the invariant characterization of homogeneous 4-spaces, J. Phys. A: Math. Gen. 36 (2003), no. 2, 427-441. MR 1960069.

[7] C. S. Gordon, E. N. Wilson, Isometry groups of Riemannian solvmanifolds, Trans. Amer. Math. Soc. 307 (1988), no. 1, 245-269. MR 0936815.

[8] M. Goze, E. Remm, Non existence of complex structures on filiform Lie algebras, Comm. Algebra 30 (2002), no. 8, 3777-3788. MR 1922311.

[9] E. Heintze, On homogeneous manifolds of negative curvature, Math. Ann. 211 (1974), 23-34. MR 0353210 
[10] E. Heintze, Riemannsche Solvmannigfaltigkeiten, Geom. Dedicata 1 (1973), no. 2, $141-147$. MR 0309012

[11] G. R. Jensen, Homogeneous Einstein spaces of dimension four, J. Differ. Geom. 3 (1969), 309-349. MR 0261487

[12] M. B. Karki, G. Thompson, Four-dimensional Einstein Lie groups, Differ. Geom. Dyn. Syst. 18 (2016), 43-57. MR 3507749

[13] J. Lauret, C. Will, Einstein solvmanifolds: existence and non-existence questions, Math. Ann. 350 (2011), no. 1, 199-225. MR 2785768.

[14] J. Milnor, Curvatures of left invariant metrics on Lie groups, Advances in Math. 21 (1976), no. 3, 293-329. MR 0425012 .

[15] A. Nijenhuis, On the holonomy groups of linear connections. IA, IB. General properties of affine connections, Nederl. Akad. Wetensch. Proc. Ser. A. 56 = Indagationes Math. 15 (1953), 233-240, 241-249. MR 0063737.

[16] G. Ovando, Invariant complex structures on solvable real Lie groups, Manuscripta Math. 103 (2000), no. 1, 19-30. MR 1794329.

[17] G. Ovando, Complex structures on $\mathbb{R} H^{4}$ and $\mathbb{C} H^{2}$, 10th School on Differential Geometry (Belo Horizonte, 1998). Mat. Contemp. 17 (1999), 237-249. MR 1787828

[18] J. E. Snow, Invariant complex structures on four-dimensional solvable real Lie groups, Manuscripta Math. 66 (1990), no. 4, 397-412. MR 1035634

S. Vukmirović

Faculty of Mathematics, University of Belgrade, Serbia

vsrdjan@matf.bg.ac.rs

M. Babić凶

Faculty of Mathematics, University of Belgrade, Serbia

marijana@matf.bg.ac.rs

A. Dekić

Faculty of Mathematics, University of Belgrade, Serbia

andrijana@matf.bg.ac.rs

Received: February 14, 2018

Accepted: December 18, 2018 